\title{
Hocus POCUS or Crucial Tool? Attitudes of General Practice Training Scheme Program Directors to Point of Care Ultrasound
}

Muiris O'Sullivan ${ }^{*}$ and Scott Walkin

Sligo Specialist Training Scheme in General Practice, Sligo, Republic of Ireland

*Corresponding author: Muiris O'Sullivan, Sligo Specialist Training Scheme in General Practice, Sligo, Republic of Ireland, Tel: 00353863146727; E-mail: muiris@hotmail.com

Received date: February 13, 2018; Accepted date: February 24, 2018; Published date: February 27, 2018

Copyright: (c) 2018 Sullivan MO, et al. This is an open-access article distributed under the terms of the Creative Commons Attribution License, which permits unrestricted use, distribution, and reproduction in any medium, provided the original author and source are credited.

\begin{abstract}
Introduction: Point of care ultrasound is used in several medical specialties but has not yet been widely adopted in primary care in Ireland. This study explored the attitudes of GP training scheme program directors towards POCUS. This included views on clinical use and implications for postgraduate GP training.

Methods: This was a mixed methods study. A survey was distributed to all GP training scheme program directors in the Republic of Ireland. The survey included questions regarding clinical use and current provision of education in point of care ultrasound to GP trainees in individual schemes. A focus group examining similar themes was also done.

Results: $50 \%$ of PDs responded to the survey. No scheme had an established training program in POCUS, and only one was considering starting one. Reasons cited included perceived lack of trainee interest, lack of faculty expertise, and lack of time and resources, including equipment. Potential uses for POCUS included basic obstetric examinations for detection of foetal heart activity, and right upper quadrant ultrasound for investigation of biliary colic. Facilitators to the use of ultrasound included rapidity of diagnosis and patient satisfaction, while barriers included lack of expertise and equipment, and concerns about clinical indemnity. The focus group corroborated these findings. PDs participating in the focus group also raise concerns about time and resource constraints both in practice and in training programs, the lack of a critical mass of expertise in the GP training community, the risk of over diagnosis, and concerns around quality assurance. The potential for "creep" effect was recognized. The group saw POCUS as having a limited potential role in a small number of clinical scenarios. Facilitators to use included patient satisfaction and increasing miniaturization and affordability of equipment.
\end{abstract}

Conclusions: There are currently no established training programs in POCUS for GP trainees in Ireland and there are no immediate plans to establish any. There is limited interest in its use in clinical practice, with multiple barriers identified.

Keywords: Point of care ultrasound (POCUS); Emergency medicine; Clinical practice; Foetal heartbeat

\section{Introduction}

Point of care ultrasound (POCUS) is now used in several medical specialties and is starting to be taught in medical schools [1-3]. POCUS involves the limited use of ultrasound at the bedside using defined protocols as a part of routine practice, and generally adheres to a "rule-in" philosophy [4]. POCUS technology is becoming cheaper and smaller, such that individual doctors can reasonably consider buying one [5]. This technological "creep" effect has been recognized by educational institutions [6], and there is increasing incorporation of POCUS curricula into undergraduate medical education and some postgraduate programs, most notably in Emergency Medicine $[7,8]$.

It is likely that we will see the use of POCUS extending into primary care [9]. There are a number of validated ultrasound protocols that can be used in this setting, including screening for left ventricular hypertrophy [10], basic obstetric ultrasound [11], and detection of deep venous thrombosis [12]. The use of POCUS by general practitioners using such protocols has been validated [13]. Should the use of this technique become commonplace, GP education in Ireland will need to incorporate POCUS training to build upon skills trainees may have acquired at undergraduate level, to teach new skills pertinent to community practice, and to certify on going professional competence in POCUS. This is no small task.

The current state of ultrasound training in GP education in Ireland is unknown, nor is the attitudes of training scheme directors towards the use of POCUS. We wanted to determine if any schemes had an ultrasound training program in place, or were intending to start one. We also wanted to find out what the facilitators and barriers to starting a training program were, and to explore the more general attitudes of program directors towards the use of POCUS in practice.

\section{Methods}

This was a descriptive study and we used two research methods, a questionnaire and a focus group.

The questionnaire was a validated instrument adapted, with permission from the authors, from a 2015 study by Hall et al. [14]. It was composed of a series of questions about current clinical practice, attitudes, and current ultrasound training programmes. Each of the 
Citation: Sullivan MO, Walkin S (2018) Hocus POCUS or Crucial Tool? Attitudes of General Practice Training Scheme Program Directors to Point of Care Ultrasound. J Gen Pract (Los Angel) 6: 351. doi:10.4172/2329-9126.1000351

Page 2 of 5

latter was a five point Likert scale ( $1=$ strongly disagree/very unlikely to $5=$ strongly agree/very likely). There were also two rank sum questions, in which respondents were asked for their first, second and third choices from a list of facilitators and barriers to POCUS use.

First ranked responses received a score of three, second a score of two and the third a score of one. Weighted scores were then listed from highest to lowest. Respondents were also encouraged to contribute qualitative "free text" comments at the end of the questionnaire. The questionnaire was distributed by post to every general practice program director and assistant program director in the Republic of Ireland. Responses were aggregated and analysed using Excel software (Microsoft Inc). We calculated proportions answering each part of the 5-point Likert scales and calculated weighted scores for the rank sum questions, from highest to lowest.

Eleven scheme directors and assistant directors were recruited for the focus group, which was conducted by the first author (MOS). We used a question guide approach. The focus group was videotaped, transcribed, and analysed (a six stage thematic analysis after Braun and Clarke) using vivo software.

\section{Results}

\section{Questionnaire}

The overall response rate was 50\% (25 questionnaires returned of 51). The questions pertained to three general themes: (Table 1).

1. Knowledge of and interest in POCUS, and current provision of POCUS training by schemes.

2. Potential uses of POCUS in trainees' future practice.

3. Perceived facilitators and barriers.

\section{Interest and background}

\begin{tabular}{|l|l|l|}
\hline Question & $\begin{array}{l}\text { Agree/ } \\
\text { Strongly agree } \\
\mathbf{n}(\%)\end{array}$ & $\begin{array}{l}\text { Neutral } \\
\mathbf{n}(\%) \\
\text { Strongly Disagree } \\
\mathbf{n}(\%)\end{array}$ \\
\hline I am familiar with the literature that supports point of care ultrasound & $5(20 \%)$ & $2(8 \%)$ \\
\hline Currently, there is interest in my scheme to train GP registrars in point of care ultrasound & $5(20 \%)$ & $6(24 \%)$ \\
\hline
\end{tabular}

Table 1: The overall response rate was 50\% (25 questionnaires returned of 51).

\begin{tabular}{|l|l|}
\hline Question & Percentage of respondents replying “yes" $\mathbf{n}(\%)$ \\
\hline We have no plans to establish ultrasound training & $24(96 \%)$ \\
\hline We are considering the addition of ultrasound training to our program & $1(4 \%)$ \\
\hline
\end{tabular}

Table 2: A single program director was considering the addition of ultrasound training to their scheme's curriculum.

Most scheme directors were not familiar with the literature around POCUS (72\%) and a minority expressed an interest in establishing an educational program in ultrasound.

No scheme had an established training program in ultrasound, and $96 \%$ had no immediate plans to start one.

A single program director was considering the addition of ultrasound training to their scheme's curriculum (Table 2).
Basic obstetric ultrasound for the detection of the foetal heartbeat, lower limb Doppler for investigation of possible deep venous thrombosis, and right upper quadrant ultrasound for the evaluation of biliary colic were all cited as possible ways in which trainees could use POCUS in their future careers.

A minority also saw a potential use for POCUS in assessment of musculoskeletal conditions, for procedural guidance such as joint injection, and for abdominal aortic aneurysm screening (Table 3).

\begin{tabular}{|l|l|l|}
\hline Question & $\begin{array}{l}\text { Unlikelyl } \\
\text { Very } \\
\text { Unlikely } \\
\text { Very Likely } \\
\mathbf{n}(\%)\end{array}$ \\
\hline Screening examinations for abdominal aortic aneurysm & $\begin{array}{l}\text { Neutral } \\
\mathbf{n}(\%)\end{array}$ \\
\hline Procedural guidance (joint injection, paracentesis, central venous line placement) & $13(44 \%)$ & $11(44 \%)$ \\
\hline Limited evaluation in trauma (FAST) & $5(36 \%)$ & $5(20 \%)$ \\
\hline Lower extremity doppler for deep venous thrombosis & $10(40 \%)$ & $17(68 \%)$ \\
\hline Right upper quadrant abdominal ultrasound for biliary colic & $12(44 \%)$ & $16(64 \%)$ \\
\hline
\end{tabular}


Citation: Sullivan MO, Walkin S (2018) Hocus POCUS or Crucial Tool? Attitudes of General Practice Training Scheme Program Directors to Point of Care Ultrasound. J Gen Pract (Los Angel) 6: 351. doi:10.4172/2329-9126.1000351

Page 3 of 5

\begin{tabular}{|l|l|l|l|}
\hline Limited echocardiogram for ejection fraction determination & $10(40 \%)$ & $5(20 \%)$ & $10(40 \%)$ \\
\hline Musculoskeletal ultrasound evaluation for tendinopathy and tears & $12(48 \%)$ & $7(28 \%)$ & $6(24 \%)$ \\
\hline Basic obstetric ultrasound for the detection of foetal heart activity & $20(80 \%)$ & $3(12 \%)$ & $2(8 \%)$ \\
\hline
\end{tabular}

Table 3: A minority also saw a potential use for POCUS in assessment of musculoskeletal conditions, for procedural guidance such as joint injection, and for abdominal aortic aneurysm screening.

\begin{tabular}{|c|c|}
\hline Perceived barriers & Rank sum \\
\hline Our scheme lacks appropriate training in performing point of care ultrasound & 29 \\
\hline GPs feel uncomfortable interpreting ultrasound images without having a radiologist available to over-read them. & 21 \\
\hline Our scheme does not have adequate access to ultrasound equipment & 18 \\
\hline Our scheme does not see a need for GPs trained in point of care ultrasound & 17 \\
\hline Ultrasound examinations are too time consuming to be done in a busy surgery & 16 \\
\hline The time GPs spend performing ultrasound examinations may not be reimbursed. & 13 \\
\hline There is no time in our current curriculum to add ultrasound training & 10 \\
\hline Clinical indemnity issues & 6 \\
\hline Students and trainees are uninterested in learning point of care ultrasound & 3 \\
\hline Clinic or hospital system policies do not permit GPs to use ultrasound in a meaningful way. & 3 \\
\hline Other: where do we find the time with all the chronic disease coming out from hospitals? & 3 \\
\hline Other: who pays for the machine and training? & 3 \\
\hline Other: we haven't discussed how we might bring it into training & 2 \\
\hline Other: patients' perception and expectation of ultrasound & 2 \\
\hline Other: I do not know if there is data to support the use of POCUS in primary care & 2 \\
\hline There are insufficient data to prove that point of care ultrasound improves patient outcomes in the ambulatory setting. & 1 \\
\hline Perceived benefits and facilitators & Rank sum \\
\hline A diagnosis is made more rapidly when the GP performs the examination at the point of care & 41 \\
\hline Point of care ultrasound use by GPs has the potential to save substantial health care costs & 27 \\
\hline Point of care ultrasound use by GPs has the potential to significantly improve patient outcomes & 24 \\
\hline Patients prefer having the examination done by their own GP & 17 \\
\hline Addition of point of care ultrasound can provide additional revenue to a GP's practice & 13 \\
\hline Medical students and residents are eager to further their skills in point of care ultrasound & 1 \\
\hline Other: GPs would get increased professional satisfaction from adding this investigation to their "tool box" & 1 \\
\hline
\end{tabular}

Table 4: Perceived facilitators and barriers.

Schemes felt they lacked the appropriate training to provide education in POCUS and also cited resource constraints such as a lack of equipment and teaching time. Respondents expressed discomfort in interpreting images without having a radiologist available to over read them. Most commonly cited facilitators to the use of POCUS included more rapid diagnosis, the potential to reduce healthcare costs, and the potential to improve patient outcomes and patient satisfaction (Table $4)$.

\section{Qualitative questionnaire data}

"I think GPs will have a special interest or skill in this area but not applicable to all GP in current model."

"This could be a skill best obtained post-graduation from GP training, learning what you don't know in the first few years while developing a subspecialty." 
“Time during consultation is very limited."

"GPs would get increased professional satisfaction from adding this investigation to their "tool box."

"Where do we find the time with all the chronic disease coming out from hospitals?"

\section{Focus group}

The focus group examined POCUS from both the perspective of use in clinical practice, and the provision of education in its use to GP trainees.

\section{Clinical use - barriers}

The group identified a number of barriers to use. These included clinician-specific factors. Some of the participants felt they lacked appropriate time, training, and knowledge to use POCUS in practice, and felt that it constituted a specialist skill as opposed to that of a generalist. viz

"I think it's really interesting but I think it's high risk ... there are a number of areas that can trip people up ... you can't do everything, so I think it will remain niche. I have no more time to devote to something like this, even though I'd love to do it" (FG4)

"You'd need a lot more training" (FG6)

Some participants also expressed fears around overdiagnosis, underdiagnosis, and potential for litigation

"The fear, you know, of the risk of missing something. That fear of missing stuff is built into all our daily work" (FG10)

"I can see my medical council thing now, and I can see the headlines in the Irish Independent" (FG2)

"One of the things we constantly give out about is the overuse of imaging in medicine ... we're trying to get away from that" (FG4)

Some members of the group felt it might be applicable for some very specific indications

"We could use it perhaps for some very specific indications, like confirming that the mirena is in the uterine cavity" (FG3

This led on to a broader discussion around the philosophy of use of POCUS in primary care, with some participants expressing concern about using a "rule-in" philosophy.

"But the nature of general practice is that you rule out rather than rule in .... it's completely different in the emergency department." (FG8)

There were also concerns about patient expectation where POCUS is employed

"Like lots of other things we do, never mind just dealing with demand, we would create a new demand, more work that we already can't do." (FG12)

Participants also outlined practical reasons why they might not use POCUS, specifically time, manpower, and resource constraints. This included the initial investment cost in the purchase of equipment, the opportunity cost of scanning time as related to consultation time, and a lack of capacity in the health service to facilitate a system of "backup" reporting from hospital based radiology.
"Just that manpower thing is a huge ... it's the resource that we're most short of ... and who's going to pay for it?" (FG7)

"We've so much to do now particularly with multimorbidity ... I'm not sure if I see this fitting in. We have to look at the resource implications - time and money"' FG2

"You'd still need loads of layers of backup I think ... I worked in Australia and there's tremendous backup ... you send it off, and half an hour later you get a phone call from a radiologist in the hospital who can talk you through it." (FG11)

Participants were also concerned about the possibility of patients being selected out for ultrasound on the basis of their ability to pay.

"Like a lot of medicine, is it economic apartheid ... if you're rich and have money then I'll do your ultrasound for you here no problem, go through that door." (FG2)

Participants also identified the potentially divisive nature of the technology within the profession, and concerns about regulation of equipment and quality assurance.

"It could be divisive though, in a practice, couldn't it, if you're the crusty old GP who doesn't have a scanner and still uses a Pinard maybe, and the young trendy doc has one!" (FG3)

"There isn't a quality assurance standard that we can have confidence in to use this equipment" (FG8).

\section{Clinical use - facilitators}

Participants felt both clinician and patient satisfaction might facilitate the use of POCUS, especially in resource-poor areas.

"There are certainly parts of Ireland where you might not be geographically remote but you might be temporally remote from getting a scan" (FG3).

"The experience I had is as a patient of near patient ultrasound ... the enormous satisfaction in having someone do it while you wait" (FG10).

Cheaper and smaller equipment was also viewed as a potential facilitator. Participants also identified the potential for a "creep" effect with increasing affordability, though this was not viewed a universally positive development.

"They're becoming more portable ... iPhone size" (FG1).

"I was thinking it'd become almost like a practice 'toy' ... People would start playing with it" (FG11).

\section{Training in POCUS}

The group identified a number of practical barriers to incorporation of POCUS education into GP training at the present time, including time and resource constraints and a lack of a critical mass of expertise within the profession to teach it.

"I think in a world where the core activities ... were well resourced, and the basic activities were taken care of and we were happy ... that we could do those well, you would have the energy and enthusiasm to go after more activity but ... it's just so far away from where we are right now" (FG3) 
Page 5 of 5

"I'm not sure that we have the actual mass, the numbers that would be interested in this that the ICGP would be able to resource courses in it" (FG2).

Most participants felt POCUS did not constitute a core competency and that starting a teaching program in it might distract from more important issues. Participants were also keen for trainees to be aware of their limitations in practice.

"What we don't want is GP trainees going through a four year scheme saying well, I'm doing the dermatology, I'm doing this and that, because the reality of the matter is that we want them to do GP training and come out competent GPs, we have to be very careful to avoid that ... You can't be doing two things at the same time" (FG12).

"We teach our graduands an awareness of their limitations and a strong ethical sense of when they're pushing the envelope" (FG3).

"I think our job is to produce rounded GPs and increasingly we need them to know what they don't know" (FG2).

\section{Discussion}

The use of point of care ultrasound appears to be in its infancy in the case of Irish general practice. There are no immediate plans at a national or regional level to establish a training programme in it. None of the doctors surveyed or interviewed used it in clinical practice, and did not feel they had sufficient expertise to teach it. Respondents identified some potential uses such as basic obstetric ultrasound; lower limb doppler for DVT, and right upper quadrant ultrasound for biliary colic.

We identified multiple barriers to the use of POCUS in clinical practice, and to its introduction into GP training in Ireland. Barriers included a lack of expertise and equipment, concerns about clinical indemnity, systems of backup, diagnostic accuracy, and time and resource constraints. Facilitators included rapidity of diagnosis, and patient and physician satisfaction.

Similar barriers were identified with regard to the introduction of POCUS education in the context of GP training. Again, a lack of time, resources, and expertise were cited, along with concerns about diagnostic accuracy, and quality assurance. POCUS was not regarded as a core clinical skill. PDs outlined the time and resource limited nature of general practice training in Ireland, and most felt that taking on additional activity was not possible at the present time.

The study was limited by its small size and response rate of $50 \%$. Triangulation of data using multiple methods was, to an extent, achieved, but on one focus group was carried out. On the other hand, this was the first study carried out in Ireland on the subject of POCUS in GP training and there was broad agreement between the questionnaire and focus group data.

POCUS presents a problem for postgraduate GP training. Medical schools are already beginning to incorporate POCUS into their curricula. We are likely to see increasing demand for POCUS from both trainees and patients, particularly with cheaper and smaller technology. This study demonstrates that GP training in Ireland is not yet prepared for the consequences of this technological advance and its implications for clinical practice and training. This could give rise to a divide within the discipline - GPs with ultrasound skills and GPs without - with implications for patient care, patient demand, and professional relationships. In this eventuality, training might be established, but in a reactive fashion. A planned, prospective approach is, to our view, preferable, and requires discussion and debate within the GP training community in Ireland, possibly in collaboration with undergraduate medical education and other medical specialities where POCUS training has already been established. There is also a need for further studies examining the attitudes of the wider GP community. POCUS presents both threats and opportunities, and requires a collaborative response to establish the practicalities and philosophies of it use in the future.

\section{References}

1. Hoppmann RA, Rao VV, Poston MB, Howe DB, Hunt PS, et al. (2011) An integrated ultrasound curriculum (iUSC) for medical students: 4-year experience. Crit Ultrasound J 3: 1-12.

2. Nelson BP, Hojsak J, Rossi ED, Karani R, Narula J (2016) Seeing is believing: Evaluating a point-of-care ultrasound curriculum for 1st-Year medical students. Teach Learn Med 29: 85-92.

3. Day J, Davis J, Riesenberg LA, Heil D, Berg K, et al. (2015) Integrating sonography training into undergraduate medical education: A study of the previous exposure of one institution's incoming residents. J Ultrasound Med 34: 1253-7.

4. Genc A, Ryk M, Suwała M, Żurakowska T, Kosiak W (2016) Ultrasound imaging in the general practitioner's office - a literature review. J Ultrason 16: 78-86.

5. Bornemann P, Bornemann G (2014) Military family physicians perceptions of a pocket point-of-care ultrasound device in clinical practice. Mil Med 179: 1474-7.

6. Bhagra A, Tierney DM, Sekiguchi H, Soni NJ (2016) Point-of-care ultrasonography for primary care physicians and general internists. mayo found med educ res n Mayo Clin Proc 91: 1811-1827.

7. Fischer LM, Woo MY, Lee AC, Wiss R, Socransky S, et al.(2015) Emergency medicine point-of-care ultrasonography: A national needs assessment of competencies for general and expert practice. CJEM 17: 74-88.

8. Atkinson P, Bowra J, Lambert M, Lamprecht H, Noble V, et al. (2015) International federation for emergency medicine point of care ultrasound curriculum. CJEM 17: 161-70.

9. Woo MY, Nussbaum C, Lee AC (2009) Emergency medicine ultrasonography: National survey of family medicine-emergency medicine program directors. Can Fam physician Médecin Fam Can 55: 1010-1.e1-4.

10. Stoica R, Heller EN, Bella JN (2011) Point-of-care screening for left ventricular hypertrophy and concentric geometry using hand-held cardiac ultrasound in hypertensive patients. Am J Cardiovasc Dis 1: 11925.

11. Moore CL, Copel JA (2011) Point-of-care ultrasonography. N Engl J Med 364: 749-757.

12. Lewiss RE, Kaban NL, Saul T (2013) Point-of-care ultrasound for a deep venous thrombosis. Glob Heart 8: 329-333.

13. Lindgaard K, Riisgaard L (2017)'Validation of ultrasound examinations performed by general practitioners'. Scand J Prim Health Care 35: 256261.

14. Hall JW, Holman H, Bornemann P, Barreto T, Henderson D, et al. (2015) Point of Care Ultrasound in Family Medicine Residency Programs: A CERA Study. Fam Med 47: 706-11. 